American Journal of Immunology 1 (2): 84-86, 2005

ISSN 1553-619X

(c) 2005 Science Publications

\title{
Comparison of Probability Curves Obtained from 2 Quantitative Allergen-specific IgE Assays: FEIA and 3gAllergy ${ }^{\mathrm{TM}}$
}

\author{
T.M. Li, T Chuang, S.M. Li and D. Hovanec-Burns \\ Invitrogen, Carlsbad, CA and Diagnostic Products Corporation, Allergy and Immunology \\ Los Angeles, CA
}

\begin{abstract}
The validity and benefit of probability curves in allergen-specific $\operatorname{IgE}$ (sIgE) testing and their applicability to more than a few allergens are still pending. One study ${ }^{[1]}$ presented probability curves for 4 food allergens and proposed decision points to rule out the need for food challenges in his clinic. Since then, such cutoffs were reported by Boyano-Martinez ${ }^{[2]}$ to be dependent on population, age, allergen and disease. A recent article by Söderström ${ }^{[3]}$ extended the use of probability curves to 8 inhalant allergens. The results were allergen dependent and site specific. None of the studies, however, has addressed the potential impact of IgE specific for cross-reacting carbohydrate determinants (CCD) on these probability curves ${ }^{[4]}$. In this study, we use logistic regression model to compare the probability curves for food and inhalant allergens using two quantitative sIgE assays; an FEIA (Pharmacia CAP) and DPC's automated chemiluminescent IMMULITE® 2000. The FEIA yields explicit results down to $0.35 \mathrm{kU} \mathrm{L}^{-1}$; the "third-generation" IMMULITE 2000 has an analytical sensitivity of $0.1 \mathrm{kU} \mathrm{L}^{-1}$ and provides precise quantification down to $0.2 \mathrm{kU} \mathrm{L}^{-1}$. In previous studies ${ }^{[6,7]}$, patients with suspected IgE-mediated allergy were evaluated according to skin prick tests or food challenges positive or negative allergen-specific diagnosis. Logistic regression was performed using the online regression analysis facility at: http://members.aol.com/johnp71/logistic.html, which is part of the Interactive Statistical Calculations web site. For inhalant allergens, almost identical overall probability curves were obtained for the FEIA and IMMULITE 2000 methods. For food allergen, similar probability curves were obtained with the two methods. The probability curves were allergen dependent. The FEIA and IMMULITE 2000 methods gave similar allergen-dependent profiles, however and in one case (W6), both methods yielded identical probability curves. The present study demonstrates that the 2 sIgE assays yield comparable probability curves for the inhalant and food allergens tested. Because the FEIA method is unable to yield explicit results below $0.35 \mathrm{kU} \mathrm{L}^{-1}$, the probability curve generated is necessarily truncated at this level. Methods for $\operatorname{sIgE}$ measurement that allow for extending probability curves below $0.35 \mathrm{kU} \mathrm{L}^{-1}$ can also assess assay performance more definitively at the traditional $0.35 \mathrm{kU} \mathrm{L}^{-1}$ cutoff. Thus the third-generation IMMULITE 2000 is a better tool for defining probability curves, as the applicability of such curves in allergy testing and the potential impact of IgE to $\mathrm{CCD}$ become well understood.
\end{abstract}

Key words: Fluorescent enzyme immunoassay, IMMULITE ${ }^{\circledR} 2000$ THIRD Generation Allergenspecific IgE, logistic regression, odds ratios, probability curves

\section{INTRODUCTION}

Sampson ${ }^{[1]}$ presented probability curves for four food allergens and proposed decision points, which could be used to eliminate the need for double-blind, placebo-controlled food challenges in his clinic. In his report, the curves illustrate graphically that even a low concentration of food-specific IgE antibodies might be associated with a risk of clinical reactivity. Since then, other investigators $^{[2]}$ have concluded that cutoff levels, however, depended on population, age, allergens and diseases. Söderström et al. ${ }^{[3]}$ extended the concept of probability curves to inhalant allergens. His study on eight allergens evaluated at four separate clinics deals with the question whether high risk was associated with higher levels of sIgE and whether quantitative results were more useful than qualitative (positive/negative) determinations. He speculated that the concept of probability for an allergic reaction or positive clinical allergy conclusion would be a better diagnostic tool compared with the traditional qualitative measures including class correlation. None of the studies, however, has addressed the potential impact of $\operatorname{IgE}$ specific for cross-reacting carbohydrate determinants (CCD) on these probability curves ${ }^{[4]}$.

The Söderström study $^{[3]}$ has led to many unanswered questions ${ }^{[5]}$. The implications of obtaining different probability curves among different clinics for the same allergens are not clear. Factors affecting the shapes of the curves are also not entirely known or

Corresponding Author: T M Li, Invitrogen, Carlsbad, CA and Diagnostic Products Corporation, Allergy and Immunology, Los Angeles, CA 
explained. For instance, why are the curves for D1 and E1 so different at the four clinics?

In previous studies ${ }^{[6,7]}$, patients with suspected IgEmediated allergy were evaluated according to skin prick tests and food challenges, respectively. The objective of our study was to apply the logistic regression model in order to compare the probability curves for one food (F2), two insects (I1 and I3) and seven inhalant (D1, D2, E1, G3, G6, T3 and W6) allergens, obtained from two quantitative sIgE methodologies: FEIA and IMMULITE 2000 Third Generation, (3gAllergy).

\section{MATERIALS AND METHODS}

Logistic regression was performed using the online regression analysis facility at: http://members.aol.com/johnp71/logistic.html, which is part of the Interactive Statistical Calculations web site.

For the two insects and seven inhalant allergens, we used skin prick tests ${ }^{[6]}$ in clinic 1 as the true status. For F2, we used food challenges in clinic $2^{[7]}$ as the true status. The same blood samples were subject to FEIA and $3 g$ Allergy analysis.

\section{RESULTS}

In the study performed at clinic 1 , almost identical overall probability curves (Fig. 1) were obtained for the FEIA and 3gAllergy methodologies, using the two insects and seven inhalant allergens with $\mathrm{N}=766$ results. Overall model fit gave Chi Square of 308.4 and 253.0, respectively with $\mathrm{df}=1$ and $\mathrm{p}=0.0001$.

Table 1: Comparison of odds ratios between the 2 methodologies

\begin{tabular}{llll}
\hline Odds Ratios & FEIA & Immulite 2000 & Difference \\
\hline D1 & 1.0575 & 1.0738 & 0.0163 \\
W6 & 2.2821 & 2.2489 & 0.0332 \\
ALL & 1.2964 & 1.1829 & 0.1135 \\
E1 & 1.1856 & 1.0566 & 0.129 \\
D2 & 1.8879 & 1.6615 & 0.2264 \\
F2 & 1.4072 & 1.1047 & 0.3025 \\
G6 & 1.7076 & 1.2677 & 0.4399 \\
G3 & 1.7202 & 1.2015 & 0.5187 \\
I3 & 2.6032 & 2.0257 & 0.5775 \\
I1 & 1.1359 & 3.4914 & 2.3555 \\
T3 & 2.0204 & 4.582 & 2.5616 \\
\hline
\end{tabular}

For the F2 food allergen study done in clinic 2, similar F2 probability curves (Fig. 2) were obtained for the FEIA and 3gAllergy methodologies: N=31 results. Overall model fit gave Chi Square of 19.1 and 17.1, respectively with $\mathrm{df}=1$ and $\mathrm{p}=0.0001$.

In agreement with Söderström's report ${ }^{[3]}$, probability curves are allergen dependent. The two FEIA and 3gAllergy methodologies, however gave similar allergen dependent profiles (Fig. 3 and 4). With certain allergens, the 2 methodologies can yield identical (W6) probability curves (Fig. 5).

According to Gallagher ${ }^{[8]}$, a positive ratio of 1 to 2 alters disease probability by a small and clinically

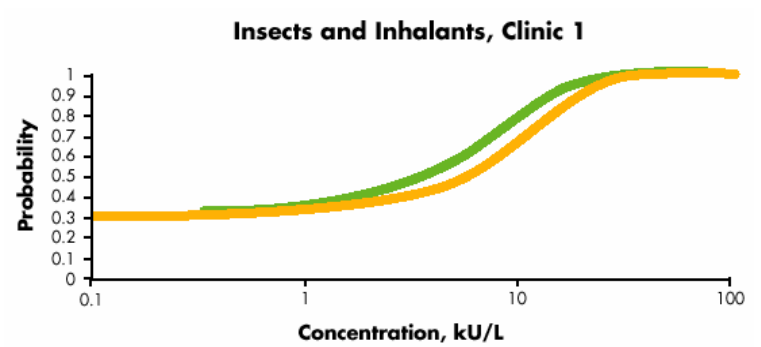

Fig. 1:Probability of receiving positive diagnosis at a given sIgE concentration for the 9 insect and inhalant allergens at clinic 1. Blue denotes FEIA methodology and magenta denotes 3gAllergy methodology

F2, Clinic 2

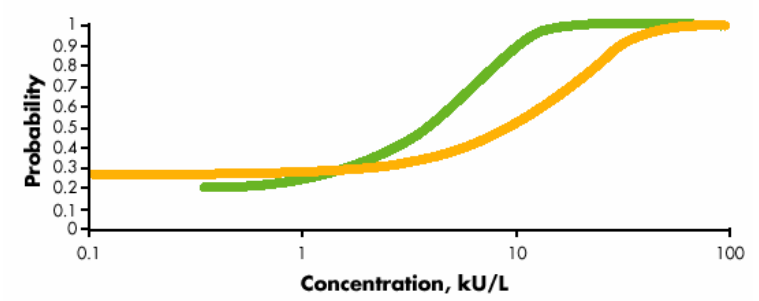

Fig. 2: Probability of receiving positive diagnosis at a given sIgE concentration for the F2 cow's milk allergen at clinic 2. Blue denotes FEIA methodology and magenta denotes 3gAllergy methodology

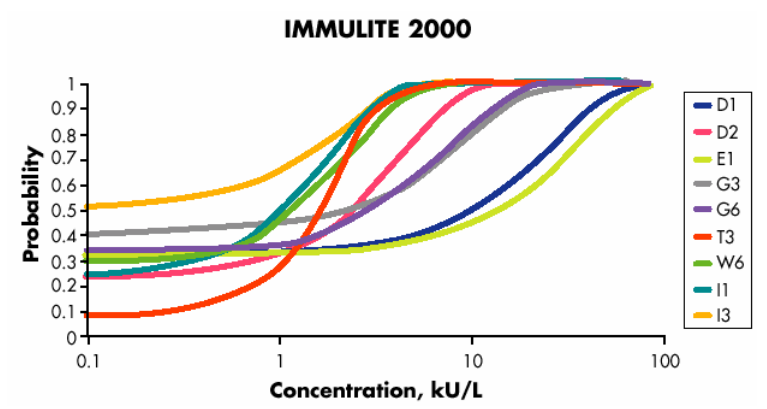

Fig. 3: Probability of receiving positive diagnosis at a given sIgE concentration for D1, D2, E1, G3, G6, T3, W6, I1 and $\mathrm{I} 3$ allergen at clinic 1 using the $3 \mathrm{~g}$ Allergy methodology

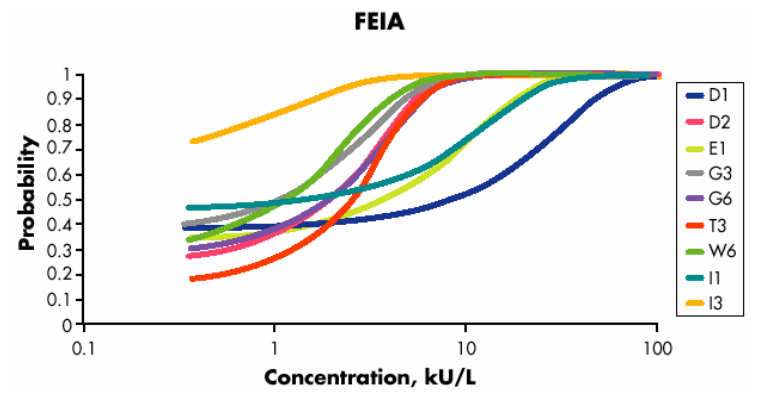

Fig. 4:Probability of receiving positive diagnosis at a given sIgE concentration for D1, D2, E1, G3, G6, T3, W6, I1 and I3 allergen at clinic 1 using the FEIA methodology 


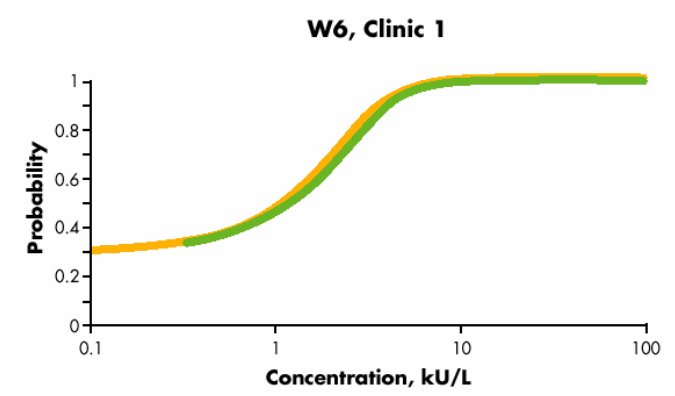

Fig. 5: Probability of receiving positive diagnosis at a given $\mathrm{sIgE}$ concentration for the W6 allergen at clinic 1. Blue denotes FEIA methodology and magenta denotes 3gAllergy methodology

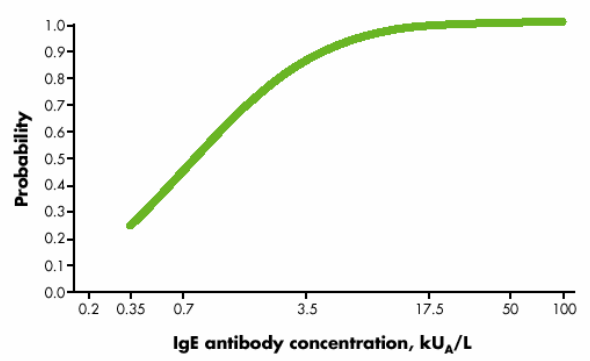

Fig. 6: From Soderstrom (2003) with permission from Blackwell Publishing: Theoretical probability curve of probability to show symptoms as a function of $\mathrm{sIgE}$ concentration

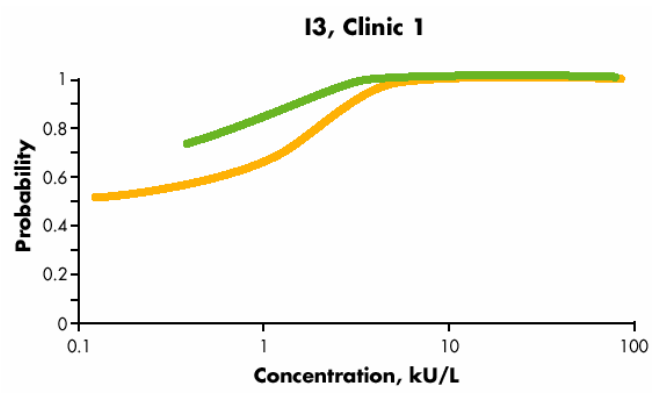

Fig. 7: Probability of receiving positive diagnosis at a given sIgE concentration for the I3 allergen at clinic 1. Blue denotes FEIA methodology and magenta denotes 3gAllergy methodology

insignificant degree. Positive ratios greater than 10 may have substantial impact on clinical decision making through meaningful revision of disease probability. Table 1 indicates there are no significant differences in the odds ratios among all of the 10 allergens evaluated.

\section{CONCLUSION}

This study demonstrates that probability curves for the two insects, seven inhalant allergens and one food allergen generated via two quantitative sIgE assays are comparable. The FEIA and 3gAllergy probability curves give odds ratios that differ by no clinical significance.
Because the FEIA methodology uses $0.35 \mathrm{kU} \mathrm{L}^{-1}$ as cut off, the probability curve generated begins at $0.35 \mathrm{kU} \mathrm{L}^{-1}$ (Fig. 6). Söderström ${ }^{[3]}$ commented that different slopes and intercepts in probability curves may indicate different identification patterns of symptoms; a steep curve would indicate symptoms easily linked to an allergen even with low levels of $\mathrm{IgE}$ antibodies. A more flat curve in contrast, would indicate a difficulty to link even high levels of $\mathrm{IgE}$ antibodies as the trigger of the symptoms. Probability curves extending below 0.35 can provide a more complete curve profile (the I3 probability curves in Fig. 7) and can be useful for the assessment of the true slope and intercept. Thus the 3gAllergy methodology which provides precise quantification down to $0.2 \mathrm{kU} \mathrm{L}^{-1}[9,10]$ can offer a better tool in defining the probability curve profile.

\section{REFERENCES}

1. Sampson, H.A., 2001. Utility of food-specific IgE concentrations in predicting symptomatic food allergy. J. Allergy Clin. Immunol., 07: 891-6.

2. Boyano, M.T., C. Garcia-Ara, J.M. Diaz-Pena, F.M. Munoz, G. Garcia Sanchez and M.M. Esteban, 2001. Validity of specific IgE antibodies in children with egg allergy. Clin. Exp. Allergy, 31: 1464-9.

3. Söderström, L., A. Kober, S. Ahlstedt, H. de Groot, C.E. Lange, R. Paganelli et al., 2003. A further evaluation of the clinical use of specific $\operatorname{IgE}$ antibody testing in allergic diseases. Allergy, 58: 921-8.

4. Mari, A., 2002. IgE to cross-reactive carbohydrate determinants: analysis of the distribution and appraisal of the in vivo and in vitro reactivity. Intl. Arch. Allergy Immunol., 129: 286-95.

5. Malandain, H., 2004. The clinical use of specific IgE antibody testing - a further word of caution [letter]. Allergy, 59: 359-60; author reply 360-1.

6. Markus, O., S. Weissenbacher, J. Rakoski and J. Ring, 2005. Allergen-specific IgE measured by a continuous random-access immunoanalyzer: interassay comparison and agreement with skin testing.

Clin. Chem., 51: 1241-1249.

7. Prates, S., 2003. IMMULITE 2000 and cow's milk allergy: a clinical perspective. In: Diagnostic Products Corporation (DPC). Proc. DPC's Intl. Allergy Conf., Jun. 6, Paris, France.

8. Gallagher, E.J., 1998. Clinical utility of likelihood ratios. Ann. Emerg. Med., 31: 391-7.

9. Li, T.M., T. Chuang, S. Tse, D. Hovanec-Burns, A.S. El Shami, 2004. Development and validation of a third generation allergen-specific IgE assay on the continuous random access IMMULITE 2000 analyzer. Ann. Clin. Lab. Sci., 34: 67-74.

10. Li, T.M. et al., 2005. Performance validation of a third generation allergen-specific IgE assay in the clinical laboratory: Interlaboratory and intermethod comparison. Clin. Chim. Acta, 361: 199-205. 